\title{
COMPARAÇÃO ESTRUTURAL ENTRE FLORESTA MANEJADA E NÃO MANEJADA NA COMUNIDADE SANTO ANTÔNIO, ESTADO DO PARÁ
}

\author{
STRUCTURAL COMPARISON BETWEEN MANAGED AND NON-MANAGED FOREST IN \\ SANTO ANTONIO COMMUNITY, PARÁ STATE
}

\author{
Diego dos Santos Vieira ${ }^{1}$ João Ricardo Vasconcellos Gama ${ }^{2}$ Renato Bezerra da Silva Ribeiro ${ }^{3}$ \\ Lucas Cunha Ximenes $^{1}$ Viviane Vasconcelos Corrêa ${ }^{1}$ Adriana Ferreira Alves ${ }^{1}$
}

\section{RESUMO}

O objetivo deste trabalho foi analisar e comparar a composição florística e estrutura horizontal da floresta manejada (FM) e da floresta não manejada (FNM). Utilizou-se amostragem casual estratificada com 12 parcelas na FM e 12 na FNM. Em parcelas de 28 x 350 m mensuraram-se todos os indivíduos com circunferência a altura do peito (CAP) maior ou igual a $31,4 \mathrm{~cm}$ e menor do que $94,2 \mathrm{~cm}$ (nível I de inclusão), subparcelas de 28 x 100 m, os indivíduos com 94,2 cm $\leq$ CAP $<157,1 \mathrm{~cm}$ (nível de inclusão II), subparcelas de 28 x 250 m e indivíduos com CAP $\geq 157,1 \mathrm{~m}$ (nível de inclusão III). Em ambas as florestas foram encontradas 216 espécies, distribuídas em 44 famílias botânicas. A FNM apresentou maior índice de diversidade de Shannon-Weaver $\left(\mathrm{H}^{\prime}\right)\left(\mathrm{H}^{\prime}=4,47\right)$. A composição florística, na $\mathrm{FM}$, não sofreu alterações significativas durante o manejo empresarial realizado. Entretanto, houve alterações significativas de riqueza e área basal entre FM e FNM devido à colheita realizada que implicou na retirada de indivíduos em classes comerciais e também ao dano causado às árvores remanescentes. As espécies de maior importância ecológica em FNM foram Rinorea guianensis Aubl (Violaceae), Pouteria bilocularis (H. Winkler) Baehni (Sapotaceae) e Sclerolobium paniculatum Vogel (Fabaceae). Em FM foram Rinorea guianensis Aubl (Violaceae), Manilkara huberi (Ducke) Chevalier (Sapotaceae) e Eschweilera coriacea (DC.) S.A. Mori (Lecythidaceae). Para o período considerado, a área basal parece estar se recuperando adequadamente.

Palavras-chave: fitossociologia; manejo florestal; Amazônia

\begin{abstract}
The aim of this study was to analyze and to compare the floristic composition and horizontal structure of managed forest (FM) and unmanaged forest (FNM). A stratified random sampling was used with 12 plots in the FM and 12 plots in the FNM. In plots of $28 \times 350 \mathrm{~m}$, all individuals with circumference at breast height (CAP) greater than or equal to $31.4 \mathrm{~cm}$ and smaller than $94.2 \mathrm{~cm}$ (inclusion level I) were measured, plots of $28 \times 100 \mathrm{~m}$, individuals with $\leq 94.2 \mathrm{~cm}$ CAP $<157.1 \mathrm{~cm}$ (inclusion level II), plots of $28 \times 250 \mathrm{~m}$ individuals with $\mathrm{CAP} \geq 157.1 \mathrm{~m}$ (inclusion level III). In both forests 216 species were found, distributed in 44 botanical families. The FNM had a higher diversity index of Shannon-Weaver $\left(\mathrm{H}^{\prime}\right)\left(\mathrm{H}^{\prime}=4.47\right)$. The floristic composition, in the FM did not change significantly during the forest management. However, there were significant changes in richness and basal area between FM and FNM because of the harvesting done which resulted in the removal of individuals in commercial classes and also to the damage caused

1 Engenheiro Florestal, Participantes do Grupo de Pesquisa em Manejo e Ecossistemas Amazônicos do Instituto de Biodiversidade e Florestas, Departamento de Engenharia Florestal, Universidade Federal do Oeste do Pará, Rua Vera Paz, s/n, CEP 68035-110, Santarém (PA), Brasil. diegovieir4@gmail.com/coldlucas@hotmail.com/ viviane-correa@hotmail.com/f.sralves18@gmail.com

2 Engenheiro Florestal, Dr., Professor Adjunto I da Universidade Federal do Oeste do Pará, Instituto de Biodiversidade e Florestas, Departamento de Engenharia Florestal, Grupo de Pesquisa em Manejo e Ecossistemas Amazônicos, Rua Vera Paz, s/n, CEP 68035-110, Santarém (PA), Brasil. jrvgama@gmail.com

3 Engenheiro Florestal, Mestrando do Programa de Pós-Graduação em Ciência Florestal, Universidade Federal de Viçosa, Departamento de Engenharia Florestal, Av. Peter Henry Rolfs, s/n, CEP 36570-000, Viçosa (MG), Brasil. Bolsista CAPES.forest_engineer@gmail.com
\end{abstract}

Recebido para publicação em 7/02/2012 e aceito em 21/05/2013 
to the remaining trees. The most important species in ecological FNM were RinoreaguianensisAubl (Violaceae), Pouteriabilocularis (H. Winkler) Baehni (Sapotaceae) and Sclerolobiumpaniculatum Vogel (Fabaceae). In FM, were RinoreaguianensisAubl (Violaceae), Manilkarahuberi (Ducke) Chevalier (Sapotaceae) and Eschweileracoriacea (DC.) S.A.Mori (Lecythidaceae). For the period considered, the basal area seems to be recovering properly.

Keywords: phytosociology; forest management; Amazon forest.

\section{INTRODUÇÃO}

O Brasil apresenta uma expressiva diversidade de ecossistemas florestais, dada a sua grande área física, diversidade de climas e solos existentes em seu território (LEITÃO-FILHO, 1987). A Amazônia, como a maior extensão de floresta tropical do mundo, apresenta uma variedade de recursos naturais e inúmeras associações vegetais que crescem sob a influência de fatores ambientais intrínsecos a cada ecossistema que forma este bioma (GAMA et al, 2003).

Qualquer intervenção planejada em florestas naturais deve ser precedida de inventário minucioso, que forneça estimativas fidedignas dos parâmetros: diversidade, frequência, densidade, dominância, distribuições diamétrica e espacial das espécies, bem como os valores ecológicos, econômico e social das espécies (SOUZA et al., 2006).

A composição florística e a estrutura da floresta são aspectos que devem ser considerados no planejamento e execução do manejo florestal para condução da floresta a uma estrutura balanceada e na escolha de práticas silviculturais adequadas para o seu melhor crescimento. Conforme Alencar (1988), o estudo da composição florística é de fundamental importância para o conhecimento da estrutura da vegetação, possibilitando informações qualitativas e quantitativas sobre a área em estudo.

O presente estudo teve o objetivo de analisar a composição florística e a estrutura horizontal da floresta manejada e da floresta não manejada por uma empresa florestal em áreas de assentamento rural.

\section{MATERIAL E MÉTODOS}

\section{Área de estudo}

A comunidade Santo Antônio abrange uma área de 5.012,25 ha e está localizada a $156 \mathrm{~km}$ de Santarém, no Projeto Assentamento (PA) Moju I e II, Rodovia Santarém-Cuiabá BR 163, Vicinal do Km 124. A colheita florestal realizada em 2006 na área manejada retirou em termos de volume 25 $\mathrm{m}^{3} \cdot \mathrm{ha}^{-1} \mathrm{e}$ em média, $5 \mathrm{~m}^{2}$.ha ${ }^{-1}$ de área basal.

O clima é classificado como Ami no sistema Köppen, ou seja, tropical úmido com variação térmica anual inferior a $5^{\circ} \mathrm{C}$ e temperatura média anual de $25,5^{\circ} \mathrm{C}$, temperaturas médias do mês mais frio sempre superiores a $18^{\circ} \mathrm{C}$, umidade relativa média do ar de $88 \%$ e precipitação pluviométrica anual média de $1.820 \mathrm{~mm}$ (FUNDAC, 2005).

\section{Amostragem e coleta de dados}

Empregou-se amostragem estratificada com parcelas alocadas sistematicamente de forma a reduzir a variância dentro dos estratos, captando a maior variabilidade e proporcionar uma boa estimativa da média. Utilizaram-se 24 unidades amostrais, sendo 12 em floresta manejada (FM) e 12 unidades amostrais em floresta não manejada (FNM), com distância fixa de $5 \mathrm{~km}$ entre FM e FNM. Considerou-se como floresta manejada área na qual houve interferência antrópica através do manejo empresarial, realizado em 2006 por meio de contrato empresarial, e floresta não manejada como área que não houve intervenção. As áreas possuem a mesma tipologia, Floresta Ombrófila Densa, e estão localizadas no mesmo local com diferenças de intervenção antrópica e por esse motivo foram consideradas como tratamentos diferentes.

As unidades de amostra foram de área fixa medindo $28 \mathrm{~m} \times 350 \mathrm{~m}$, onde foram mensurados todos os indivíduos com circunferência igual ou superior a $31,4 \mathrm{~cm}$, a $1,30 \mathrm{~m}$ do solo (CAP), considerando-se os seguintes níveis de inclusão e tamanhos de parcela: Nível 1 de inclusão: $31,4 \mathrm{~cm}$ $\leq \mathrm{CAP}<94,2 \mathrm{~cm}$ em subparcelas de $28 \mathrm{~m}$ x $100 \mathrm{~m}$; Nível 2 de inclusão: 94,2 $\mathrm{cm} \leq \mathrm{CAP}<157,1 \mathrm{~cm}$ em subparcelas de 28 m x 250 m; e Nível 3 de inclusão: $\mathrm{CAP} \geq 157,1 \mathrm{~cm}$; na parcela de $28 \mathrm{~m}$ x $350 \mathrm{~m}$.

Formas de utilização das espécies foram verificadas por meio de entrevistas com comunitários e classificadas nos seguintes grupos: 
madeireiro, frutífera, alimento para caça, medicinal, carvão e outros usos.

\section{Análise de dados}

A composição florística foi analisada com base na distribuição dos indivíduos em espécies e famílias. De posse da listagem de espécies, foram estimados os índices de similaridade de Sörensen, de diversidade de Shannon-Weaver, de equabilidade de Pielou, de Payandeh e o Quociente de Mistura de Jentsch, (BROWER e ZAR, 1984).

Foram consideradas espécies de baixa densidade, aquelas com menos de um indivíduo por hectare e as espécies indicadoras, aquelas que ocorreram em apenas uma das florestas estudadas (GAMA et al., 2007). A comparação do índice de Shannon-Weaver, entre as duas fitocenoses, foi realizada por meio do teste t-Student, proposto por Hutcheson (1970). Os parâmetros fitossociológicos da estrutura horizontal foram estimados conforme Mueller-Dombois e Ellenberg (1974).

As variáveis densidade, área basal, volume, riqueza e grupo de uso foram analisadas pelo teste t-Student, para amostras independentes conforme Banzatto e Kronka (1989), para testar a existência de igualdade entre as médias dos parâmetros estudados, a 5\% de probabilidade.

Para todas as variáveis analisadas, foram aplicadostestes denormalidadede Shapiro-Wilk, para averiguar se o conjunto de observações se considera proveniente de uma população com distribuição normal, e de homogeneidade de variância; quando não houve normalidade e homogeneidade aplicouse transformação logarítmica para o emprego da estatística paramétrica. A tabulação e o processamento de dados foram realizados por meio dos programas Excel 2010 e Biostat 5.0.

\section{RESULTADOS E DISCUSSÃO}

\section{Composição florística}

Foram identificadas 172 e 183 espécies, respectivamente, naFM e naFNM, sendo 31 espécies exclusivas da FM e 43 da FNM. Comparando-se esses resultados com os de outros estudos, verificouse que: em Marabá - PA, Garcia (1990) encontrou 97 espécies; na Estação Experimental de Curuá-Uma PA, Barros et al (2000) identificaram 188 espécies; na FLONA do Tapajós- PA, Espírito-Santo et al. (2005) localizaram 190 espécies; em Cachoeira
Porteira - PA, Lima Filho et al. (2004) registraram 359 espécies; na FLONA Saracá-Taquera - PA, Salomão et al. (2000) identificaram 311 espécies e Batista et al. (2011), em Santarém-Novo - PA, encontraram 51 espécies. Essas diferenças provavelmente ocorrem devido aos diferentes tamanhos de amostras, níveis, classe de solo e a situação antrópica.

As famílias com maior riqueza de espécies em ordem decrescente, na FM, foram Fabaceae (41), Sapotaceae (13), Moraceae (11), Lecythidaceae (10) e Apocynaceae (8) que, juntas, contribuem com $48,2 \%$ da riqueza local, ficando as 35 famílias restantes, responsáveis por $51,7 \%$ do total, evidenciando a baixa abundância relativa de indivíduos nestas famílias. As famílias mais importantes na FNM foram Fabaceae (49), Sapotaceae (14), Moraceae (11), Lecythidaceae (9) e Apocynaceae (7) que, juntas, representam um total de $49,2 \%$ da riqueza local, ficando 37 famílias responsáveis por $50,8 \%$ do total. Esses resultados confirmam os inventários florísticos realizados em floresta de terra firme (AMARAL et al., 2000; LIMA FILHO et al., 2001; OLIVEIRA e AMARAL, 2004), onde essas famílias destacam-se entre as dez mais diversificadas.

Foram encontradas diferenças significativas, pelo teste t-Student, entre o número total de espécies $(\mathrm{p}<0,04)$ e área basal $(\mathrm{p}<0,01)$ provavelmente devido à exploração a qual reduz somente o estoque de madeira na área manejada afetando diretamente a área basal, e também pode causar alguns danos na vegetação remanescente, pois muitas árvores remanescentes morrem ou sofrem injúrias colaborando na redução do número total de espécies. Deve ser mencionado que a redução em área basal é esperada, e encontra-se em $80 \%$ do original, tendo transcorrido apenas 6 anos, para um ciclo total de 25 anos. O índice de equabilidade de Pielou $(J)$, que permite representar a uniformidade da distribuição dos indivíduos entre as espécies, indicou que $86 \%$ da diversidade máxima foi encontrada na amostragem realizada na FM e na FNM, ou seja, não há um descompasso na relação de indivíduos $\mathrm{x}$ espécies entre as florestas,. O quociente de mistura de Jentsch $(Q M)$, que define o número de indivíduos amostrados em relação às espécies encontradas no povoamento, foi de 1:8 para as duas florestas (Tabela 1). Finol (1975) afirmou que, em florestais tropicais, o quociente de mistura seria de aproximadamente nove indivíduos por espécies, indicando alta heterogeneidade.

A similaridade florística entre FM e FNM foi 
TABELA 1: Índices de diversidade de Floresta manejada (FM) e Floresta não manejada (FNM), na Comunidade Santo Antônio, Município de Santarém, Estado do Pará, Brasil.

TABLE 1: Diversity indices of managed forest (FM) and unmanaged forest (FNM) in Santo Antônio community, municipality of Santarém, Pará state, Brazil.

\begin{tabular}{|c|c|c|}
\hline Parâmetro & FM & FNM \\
\hline Densidade absoluta $\left(\mathrm{n}^{\mathrm{o}} \text { ind.ha }{ }^{-1}\right)^{\mathrm{ns}}$ & 255 & 299 \\
\hline Área basal $\left(\mathrm{m}^{2} \cdot \mathrm{ha}^{-1}\right)^{*}$ & 18,47 & 23,12 \\
\hline Riqueza de espécies $(\mathrm{S}) *$ & 172 & 183 \\
\hline Número de espécies de baixa densidade absoluta ${ }^{\text {ns }}$ & 111 & 113 \\
\hline Número de espécies indicadoras ${ }^{\text {ns }}$ & 32 & 43 \\
\hline Diversidade máxima $\left(\mathrm{H}_{\text {máx }}\right)^{\text {ns }}$ & 5,15 & 5,21 \\
\hline Índice de Shannon-Weaver $(\mathrm{H}>)^{\mathrm{ns}}$ & 4,41 & 4,47 \\
\hline Equabilidade de Pielou (J) ns & 0,86 & 0,86 \\
\hline Coeficiente de Mistura de Jentsch $(\mathrm{QM})^{\mathrm{ns}}$ & $1: 8$ & $1: 8$ \\
\hline Índice de Sörensen & 0,79 & \\
\hline
\end{tabular}

Em que: *Diferenças significativas (p $<0,05)$, pelo teste t-Student, entre FM E FNM, ${ }^{\text {ns }}$ - não significativo a 5\% de probabilidade pelo teste $\mathrm{t}$.

alta $\mathrm{S}>0,79$, ou seja, $79 \%$ das espécies ocorreram nas duas áreas. De acordo com Kent e Coker (1992), valores iguais ou maiores a 0,5 indicam alta similaridade.

A diversidade florística foi de 4,41 e 4,47, respectivamente para FM e FNM. Segundo Knight (1975), a diversidade para florestais tropicais de terra firme varia de 3,38 a 5,85, pode-se afirmar que os ambientes florestais apresentaram alta diversidade florística. Oliveira e Amaral (2004), estudando uma floresta de vertente na Amazônia Central, encontraram $H^{\prime}=5,01$, considerando indivíduos arbóreos com DAP $>10 \mathrm{~cm}$.

\section{Padrão de distribuição espacial}

Na FM, 28,3\% das espécies apresentaram padrão aleatório, $18,5 \%$ tendência à agregação, $26,6 \%$ padrão agregado e 26,6 espécies raras. Tendência semelhante foi observada na FNM, onde $25,1 \%$ das espécies tiveram padrão aleatório, $15,8 \%$ tendência à agregação, $34,4 \%$ padrão agregado e $24,6 \%$ espécies raras. Para Nascimento et al. (2001), espécies que apresentam este tipo de padrão de distribuição necessitam de maior área de amostragem e uma distribuição de unidades amostrais para a definição de seu padrão de distribuição espacial.

\section{Estrutura horizontal}

Na FM foram estimados, considerandose DAP $\geq 10 \mathrm{~cm}, 255$ ind. ha ${ }^{-1}$ e $18,476 \mathrm{~m}^{2} \cdot \mathrm{ha}^{-1} \mathrm{de}$ área basal. Na FNM constataram-se 299 ind. ha ${ }^{-1} \mathrm{e}$ área basal de $23,127 \mathrm{~m}^{2}$.ha-1. O resultado do teste t-Student não mostrou diferenças significativas, $\mathrm{p}<0,01$ e $\mathrm{p}<0,10$, respectivamente, nas duas características.

Dentre as 172 espécies identificadas na FM, 63 apresentaram densidade absoluta (DA) igual ou superior a 1. As 9 espécies mais abundantes (DA $\geq 5$ ) foram Rinorea guianensis, Protium cf. heptaphyllum, Eschweilera coriacea, Richardella macrophylla, Pouteria bilocularis, Sclerolobium, Sclerolobium paniculatum, Protium paniculatum var. riedelianum, Nectandra spp., que, juntas, representam $31,6 \%$ da densidade absoluta total.

Dentre as 183 espécies identificadas na FNM 75 apresentaram $D A \geq 1$. As 9 espécies mais abundantes (DA $\geq 6$ ) foram Rinorea guianensis, Eschweilera coriacea, Protium cf. heptaphyllum, Inga alba, Pouteria guianensis, Sclerolobium, Franchetella anibaefolia, Protium paniculatum var. riedelianum, Manilkara huberi, que, juntas, contribuíram com 30,3\% da densidade absoluta total (Tabela 2).

Analisando as espécies de maiores valores de DA, que também apresentaram as maiores 
frequências absolutas (FA), destacaram-se, na FNM, Pouteria bilocularis, Sclerolobium paniculatum e Manilkara huberi na FNM.

$\mathrm{Na}$ FM, as 10 espécies de maior dominância absoluta $\left(\right.$ DoA $\left.\geq 0,39 \mathrm{~m}^{2} \cdot \mathrm{ha}^{-1}\right)$ foram Rinorea guianensis, Pouteria bilocularis, Sclerolobium paniculatum, Sclerolobium, Lecythis jarana, Manilkara huberi, Carapa guianensis, Chamaecrista scleroxylon, Protium cf. heptaphyllum, Geissospermum sericeum, que, juntas, representaram $32,5 \%$ da dominância total (DoT).

Na FNM, as 10 espécies de maior dominância absoluta (DoA $\geq 0,53 \mathrm{~m}^{2} . \mathrm{ha}^{-1}$ ) foram Manilkara huberi, Rinorea guianensis, Eschweilera coriacea, Sclerolobium, Pouteria bilocularis, Franchetella anibaefolia, Lecythis jarana, Sclerolobium paniculatum, Carapa guianensis, Eschweilera grandiflora, que, juntas, colaboraram com $32,1 \%$ da DoT.

TABELA 2: Estimativa dos parâmetros fitossociológicos das espécies com valor de importância (VI) $\geq$ 1,5. (I = floresta manejada, II = floresta não manejada, $\mathrm{DA}=$ densidade absoluta, em n.ha; FA = frequência absoluta, em porcentagem; DoA = dominância absoluta, em $\mathrm{m}^{2} \mathrm{ha}^{-1}$; $\mathrm{VI}=$ valor de importância, em porcentagem; $\mathrm{AG}=$ agregado; $\mathrm{TAG}=$ tendência a agregação; $\mathrm{AL}=$ aleatório).

TABLE 2: $\quad$ Estimate of phytosociological parameters of the species with $\mathrm{VI} \geq 1.5$. In which: I= managed forest; $\mathrm{II}=$ unmanaged forest; $\mathrm{DA}=$ absolute density, in ind.ha ${ }^{-1}, \mathrm{FA}=$ absolute frequency, in percentage; DoA $=$ absolute dominance in $\mathrm{m}^{2} \cdot \mathrm{ha}^{-1} ; \mathrm{VI}=$ importance value, in percentage; $\mathrm{AG}=$ clustered; $\mathrm{TAG}=$ tendency to cluster; $\mathrm{AL}=$ randomized

\begin{tabular}{|c|c|c|c|c|c|c|c|c|c|c|}
\hline \multirow{2}{*}{ Espécies } & \multicolumn{2}{|c|}{$\mathrm{P}$} & \multicolumn{2}{|c|}{ DA } & \multicolumn{2}{|c|}{ FA } & \multicolumn{2}{|c|}{ DoA } & \multicolumn{2}{|c|}{ VI } \\
\hline & I & II & I & II & I & II & I & II & I & II \\
\hline Rinorea guianensis Aubl. & $\mathrm{AG}$ & $\mathrm{AG}$ & 26,2 & 24,3 & 75 & 83 & 1,054 & 0,812 & 5,84 & 4,39 \\
\hline Pouteria bilocularis (H. Winkler) Baehni & TAG & $\mathrm{AG}$ & 6,0 & 4,3 & 100 & 92 & 0,819 & 0,643 & 2,94 & 1,97 \\
\hline Sclerolobium paniculatum Vogel & $\mathrm{AL}$ & $\mathrm{AL}$ & 5,3 & 4,8 & 100 & 92 & 0,729 & 0,540 & 2,69 & 1,88 \\
\hline Protium cf. heptaphyllum (Aubl.) Marchand & $\mathrm{AG}$ & $\mathrm{AG}$ & 10,4 & 8,8 & 67 & 67 & 0,399 & 0,352 & 2,53 & 1,90 \\
\hline Sclerolobium sp. & $\mathrm{AG}$ & AG & 5,9 & 8,1 & 67 & 75 & 0,651 & 0,733 & 2,41 & 2,42 \\
\hline Eschweilera coriácea (DC.) S.A.Mori & $\mathrm{AG}$ & $\mathrm{AG}$ & 10,3 & 12,4 & 33 & 83 & 0,385 & 0,807 & 2,27 & 3,07 \\
\hline Manilkara huberi (Ducke) Chevalier & TAG & TAG & 3,5 & 6,0 & 92 & 100 & 0,553 & 1,597 & 2,07 & 3,59 \\
\hline Richardella macrophylla (Lam.) Aubrév. & TAG & - & 6,6 & - & 83 & - & 0,255 & - & 1,89 & - \\
\hline Carapa guianensis Aubl. & $\mathrm{AG}$ & $\mathrm{AG}$ & 4,6 & 3,7 & 67 & 50 & 0,436 & 0,537 & 1,84 & 1,49 \\
\hline Geissospermum sericeum Benth. \& Hook.f. ex Miers & TAG & - & 4,0 & - & 83 & - & 0,396 & - & 1,81 & - \\
\hline Lecythis jarana (Huber \&Ducke) A. C. Smith & $\mathrm{AG}$ & TAG & 2,3 & 3,9 & 67 & 92 & 0,558 & 0,606 & 1,77 & 1,88 \\
\hline $\begin{array}{l}\text { Chamaecrista scleroxylon (Ducke) H.S.Irwin \& } \\
\text { Barneby }\end{array}$ & $\mathrm{AG}$ & - & 4,7 & - & 58 & - & 0,415 & - & 1,76 & - \\
\hline Nectandra sp. & $\mathrm{AL}$ & - & 5,0 & - & 92 & - & 0,208 & - & 1,65 & - \\
\hline $\begin{array}{l}\text { Protium paniculatum var. riedelianum (Engl.) } \\
\text { D.C.Daly }\end{array}$ & $\mathrm{AG}$ & $\mathrm{AG}$ & 5,1 & 6,6 & 58 & 75 & 0,315 & 0,408 & 1,63 & 1,79 \\
\hline Pouteria caimito (Ruiz \& Pav.) Radlk. & $\mathrm{AG}$ & - & 3,9 & - & 67 & - & 0,308 & - & 1,52 & - \\
\hline Franchetella anibaefolia (A.C. Smith.) Aubr. & - & $\mathrm{AG}$ & - & 7,8 & - & 67 & - & 0,619 & - & 2,17 \\
\hline Pouteria guianensis Aubl. & - & $\mathrm{AG}$ & - & 8,3 & - & 92 & - & 0,435 & - & 2,11 \\
\hline Eschweilera grandiflora (Aubl.) Sandwith & - & TAG & - & 4,5 & - & 83 & - & 0,532 & - & 1,79 \\
\hline Inga alba (Sw.) Willd. & - & $\mathrm{AG}$ & - & 8,6 & - & 58 & - & 0,304 & - & 1,76 \\
\hline Minquartia guianensis Aubl. & - & $\mathrm{AG}$ & - & 4,0 & - & 58 & - & 0,513 & - & 1,54 \\
\hline Subtotal & - & - & 104 & 116 & - & - & 7,5 & 9,4 & 34,6 & 33,7 \\
\hline Outras & - & - & 151 & 183 & - & - & 11,0 & 13,7 & 65,4 & 66,3 \\
\hline Total & - & - & 255 & 299 & - & - & 18,5 & 23,1 & 100 & 100 \\
\hline
\end{tabular}


As 10 espécies com maiores valores de importância (VI > 1,8\%) na FM foram Rinorea guianensis - seus frutos são consumidos por animais silvestres; Pouteria bilocularis - sua madeira é utilizada por serrarias e seus frutos são consumidos pela fauna; Protium cf. heptaphyllum, Eschweilera coriacea e Manilkara huberi - estas três espécies possuem uso madeireiro e os seus frutos são consumidos por animais silvestres; Richardella macrophylla - madeira usada em serrarias, uso frutífero, e utilizada para produção de carvão; Carapa guianensis - sua madeira é utilizada por madeireiras, serve de alimento para a fauna e seu óleo é utilizado na medicina caseira; Geissospermum sericeum - além de servir de alimento para animais possui propriedades medicinais; Sclerolobium paniculatum, Sclerolobium - estas duas espécies possuem usos desconhecidos. Juntas, estas espécies representam $27,1 \%$ do valor de importância.

$\mathrm{Na}$ FNM as 10 maiores espécies com maiores valores de importância (VI > 1,7\%) foram Rinorea guianense e Franchetella anibaefolia - seus frutos servem de alimento para animais silvestres; Manilkara huberi, Eschweilera coriácea, Pouteria bilocularis, Protium cf. heptaphyllum, Lecythis jarana - todas estas espécies apresentam uso direcionado para madeireiras e consumo de seus frutos por animais silvestres; Pouteria guianensis além de seus frutos serem consumidos por animais, sua madeira ser utilizada por serrarias e para produção de carvão, há também uso direcionado para construção de postes na comunidade; Sclerolobium paniculatum, Sclerolobium - estas espécies apresentaram uso desconhecido na comunidade. Estas 10 espécies contribuem com 26,2\% do valor de importância.

Dentro da avaliação do índice de valor de importância determinou-se como a principal espécie Rinorea guianensis que ocorreu entre as 10 maiores espécies com valor de importância estudado, podendo-se inferir que esta espécie apresenta o maior domínio do habitat, ou seja, é a espécie mais importante nas duas áreas estudadas.

Somada a esta, estão Pouteria bilocularis, Sclerolobium paniculatum, Protium $c f$. heptaphyllum, Sclerolobium, Eschweilera coriacea, Manilkara huberi, Richardella macrophylla, Carapa guianensis, Geissospermum sericeum, totalizando $20,45 \%$ do VI total.

Barros et al. (2000), ao analisarem a estrutura considerando indivíduos da classe de $10 \leq$ DAP $<45 \mathrm{~cm}$ de uma floresta situada em Curuá-Una, verificaram que Rinorea guianensis, Tetragastris panamensis, Eschweilerasa gotiana, Protiumsa gotianum e Mouriri sp. foram as espécies mais abundantes e com maior VI na área. No presente estudo, apenas Rinorea guianensis está entre as espécies com maior VI em uma floresta em CuruáUna.

Merecem destaque Rinorea guianensis, Pouteria bilocularis, Eschweilera coriacea e Manilkara huberi, que ocorreram entre as 10 com maiores valores de importância nas duas florestas estudadas, podendo-se inferir que essas mesmas florestas apresentaram maior domínio do habitat, ou seja, são as espécies mais importantes ecologicamente na floresta manejada e não manejada (GAMA et al, 2007).

\section{Distribuição diamétrica}

A distribuição do número de árvores por classe diamétrica, com amplitude de $10 \mathrm{~cm}$, seguiu o padrão característico das florestas inequiâneas, isto é, distribuição exponencial negativa, na forma de "J" invertido, que, segundo Amaral et al. (2000), Lima Filho et al. (2001) e Oliveira e Amaral (2004), é o comportamento típico de muitas florestas de terra firme da Amazônia Oriental. Nota-se que 81,9 e $79,6 \%$ dos indivíduos concentram-se entre o intervalo de 15 a $45 \mathrm{~cm}$, respectivamente, na FM e FNM; o que caracteriza uma floresta com árvores de pequeno porte (Figura 1).

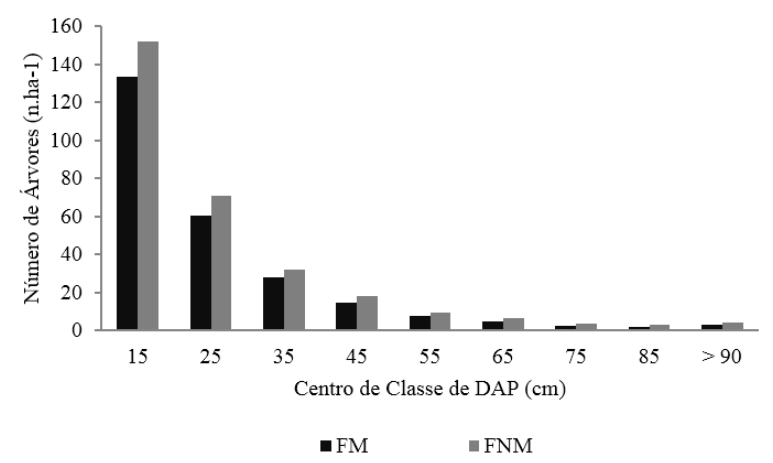

FIGURA 1: Distribuição diamétrica dos indivíduos de uma floresta ombrófila densa em duas áreas na Comunidade Santo Antônio, Município de Santarém, Estado do Pará.

FIGURE 1: Diametric distribution of the individuals in a dense ombrophylous forest in Santo Antônio community, municipality of Santarém, Pará state. 
Gonçalves e Santos (2008) em um estudo realizado na FLONA do Tapajós, também encontraram um padrão de distribuição diamétrica na forma de "J" invertido, sugerindo um balanço entre o recrutamento e mortalidade de árvores na área.

\section{CONCLUSÃO}

Houve alta similaridade (79\%) entre as espécies contidas no estrato adulto de FM e FNM, indicando que a maioria das espécies ocorre nos dois estratos;

O coeficiente de mistura (QM) foi baixo para as duas florestas, evidenciando que o manejo em ambas as áreas dever ser mais criterioso para que não haja alterações significativas em suas estruturas;

As famílias mais ricas nas duas florestas foram Fabaceae e Sapotaceae;

Na FM houve o predomínio da distribuição aleatória $(28,3 \%)$, devido ao fato de a floresta ter sofrido corte e na FNM houve predomínio do padrão de distribuição agregado $(34,4 \%)$, tal comportamento é característico de floresta não explorada;

A espécie Rinorea guianensis apresentou maior importância nas áreas, tanto na floresta explorada como na floresta não explorada, devido a isso pode tornar-se uma espécie potencial para programas ambientais voltados para reflorestamento e recuperação de áreas degrada.

Para o período considerado, a área basal parece estar se recuperando adequadamente.

\section{AGRADECIMENTOS}

Ao Conselho Nacional de Desenvolvimento Científico e Tecnológico (CNPq/UFOPA), pela bolsa concedida ao primeiro autor.

\section{REFERÊNCIAS BIBLIOGRÁFICAS}

ALENCAR, J. DA C. Metodologia para análise de vegetação. In: ENCONTRO SOBRE INVENTÁRIOS NA AMAZONIA, 1988, Manaus, Anais... Manaus, 1988. 19 p.

AMARAL, I. L.; MATOS F. D. A.; LIMA J. Composição florística e estrutural de um hectare de floresta densa de terra firme no Rio Uatumã, Amazônia, Brasil. Acta Amazonica.v. 30, n. 4, p. 377-392, 2000.

BANZATTO, D. A.; KRONKA, S. N.
Experimentação agrícola. Jaboticabal: Universidade Estadual de São Paulo. 1989. 247 p. BARROS, A.V. de; BARROS, P. L. C. de; SILVA, L. C. B. da. Análise fitossociológica de uma floresta situada em Curuá-Una - Pará. Revista de Ciências Agrárias, v. 34 n. 1, p. 9 - 36, 2000.

BATISTA, F. J. et al. Comparação florística estrutural de duas florestas de várzea no estuário amazônico, Pará, Brasil. Revista Árvore, v. 35, n. 2, p. 289-298, 2001.

BROWER, J. E.; ZAR, J. H. Field and laboratory methods for general ecology. Dubuque: W. M. C. Brow, 1984. $226 \mathrm{p}$.

ESPÍRITO-SANTO, F. D. B. et al. Análise da composição florística e fitossociológica da floresta nacional do tapajós com o apoio geográfico de imagens de satélites. Acta Amazonica. v. 35, n. 2, p. 255-173, 2005.

FINOL, U. H. La silvicultura em laOrinoquiaVenezolana. Revista Forestal Venezolana, v. 18, n. 25, p. 37 -114, 1975.

FUNDO DE DESENVOLVIMENTO E AÇÃO COMUNITÁRIA (FUNDAC). Plano de Desenvolvimento Sustentável Mojú I e II. Santarém: FUNDAC, 2005. 153 p.

GAMA, J. R. V. et al. Estrutura potencial futuro de utilização da regeneração natural de floresta de várzea alta no Município de Afuá, Estado do Pará. Ciência Florestal, v. 13, n. 2, p. 71-83, 2003.

GAMA, J. R. V.; SOUZA, A. L.; CALEGÁRIO,N.; LANA, G. C. Fitossociologia de duas fitocenoses de floresta ombrófila aberta no município de Codó, Estado do Maranhão. Revista Árvore, v. 31, n. 3, p. 465-477, 2007.

GARCIA, A. Influência de diferentes níveis de exploração florestal em uma floresta tropical na região de Marabá- PA. 1990. 149 f. Dissertação (Mestrado em Ciências Florestais) - Escola Superior de Agricultura Luiz de Queiroz, Piracicaba, 1990.

GONÇALVES, F. G.; SANTOS, J. R. Composição florística e estrutura de uma unidade de Manejo florestal sustentável na Floresta Nacional do Tapajós, Pará. Acta Amazônica, v. 38, n. 2, p. 229 244, 2008.

HUTCHESON, K. A test for comparing diversities based on the Shannon formula. Journal of Theoretical Biology, v. 29, p. 151-154, 1970.

LEITÃO FILHO, H. F. Considerações sobre a florística de florestas tropicais e sub-tropicais do Brasil. IPEF, v. 35, n.35, p. 41-46, 1987

LIMA FILHO, D. A. et al. Inventário florístico de floresta ombrófila densa de terra firme, na região 
do Rio Urucu-Amazonas, Brasil. Acta Amazonica, v. 31, n. 4, p. 565-579. 2001.

LIMA FILHO D. A. et al. Aspectos florísticos de 13 hectares da área de Cachoeira Porteira-PA. Acta Amazonica. v. 34, n. 3, p. 415-423, 2004.

MUELLER-DOMBOIS, D.; ELLEMBERG, H. Aims and methods of vegetation ecology. New York: John Wiley \& Sons, 547 p. 1974.

NASCIMENTO, A. R. T.; LONGHI, S. J.; BRENA, D. A. Estrutura e padrões de distribuição espacial de espécies arbóreas em uma amostra de Floresta Ombrófila Mista em Nova Prata, RS. Ciência Florestal, v. 11, n. 1, p. 105-119, 2001.

OLIVEIRA, A. N.; AMARAL, I. L. Florística e fitossociologia de uma floresta de vertente na Amazônia Central, Amazonas, Brasil. Acta Amazonica, v. 34, n. 1, p. 21-34, 2004.
KENT, M.; COKER, P. Vegetation description analyses. London: Behaven Press, 1992. 363 p.

KNIGHT, D.H. A phytosociological analysis of species-rich tropical forest on Barro Colorado Island, Panama. Ecological Monographs, v. 45, p. 259-28, 1975.

SALOMÃO, R. P.; ROSA, N. A.; MATOS, A. H. Estudo e monitoramento da floresta tropical primária visando a restauração da paisagem florestal em áreas degradadas da amazônia brasileira. In: SIMPÓSIO NACIONAL RECUPERAÇÃO DE ÁREAS DEGRADADAS, 4., 2000. Anais... Blumenau: SOBRADE, 2000.

SOUSA, D. R. et al. Análise estrutural em florestal ombrófila densa de terra firme não explorada, Amazônia Oriental. Revista Árvore, v. 30, n. 1, p. $75-87,2006$. 